\title{
A Pathologist's Approach to Nonimmune Hydrops
}

\author{
W. Tony Parks ${ }^{1,2}$
}

Received: 24 June 2015/ Accepted: 24 September 2015/Published online: 11 November 2015

(C) Society of Fetal Medicine 2015

\begin{abstract}
Fetal hydrops is an uncommon, but frequentlylethal, complication of pregnancy. Hydrops can result from any of diverse etiologies, although it has classically been divided into immune and nonimmune hydrops. While the etiology for many cases can be determined prenatally, pathologists are nearly always called on to identify the underlying cause for those cases that remain unexplained at the time of delivery or termination. This brief review will be confined to nonimmune hydrops, and the discussion will particularly focus on the role of the pathologist in evaluating this condition.
\end{abstract}

Keywords Nonimmune - Hydrops - Autopsy - Genetic testing · Chromosomal microarray · Lysosomal storage disorder - Anemia - Cardiac anomaly - Fetomaternal hemorrhage · Parvovirus B19

\section{Introduction}

Hydrops fetalis remains an often-devastating pregnancy complication. Hydrops is not a single disease but rather a symptom complex that arises as the final common pathway from a surprisingly large number of diverse etiologies. Diagnosis requires the detection of excess fluid in at least two fetal body compartments (ascites, pleural effusions,

W. Tony Parks

parkswa@upmc.edu

1 Departments of Pathology and Obstetrics, Gynecology and Reproductive Sciences, University of Pittsburgh School of Medicine, Pittsburgh, PA, USA

2 Department of Pathology, Magee-Women's Hospital, 300 Halket Street, Pittsburgh, PA 15213, USA pericardial effusions, or skin edema [1,2]. Polyhydramnios and a thickened placenta (on ultrasound examination) often accompany the fluid accumulations in body compartments but are not typically considered as diagnostic criteria [3, 4]. Due to a number of physiologic differences between intrauterine and extrauterine life, fluid accumulation in fetal tissues occurs more easily than in an infant or adult, and this process readily extends to the generalized edema with fluid collections in body cavities characteristic of hydrops [5]. The pathophysiologic processes underlying interstitial fluid accumulation in hydrops include (but are not limited to) cardiac dysfunction (particularly those disorders that result in increased central venous pressure), anemia (generally with high output cardiac failure), lymphatic obstruction, altered endothelial cell integrity with increased capillary permeability and hypoproteinemia (as seen with disruptions to hepatic synthetic capacity) $[5,6]$.

Hydrops has been reported to arise in 1 in 1700-3000 pregnancies [7-9]. The data underlying these estimates are suboptimal, however, and newer, more definitive measurements of prevalence rates are needed [6]. Hydrops occurred more frequently in the past due to Rhesus blood group antigen isoimmunization, with isoimmunization representing the most common etiology. The development of anti-D gamma globulin (RhoGAM) has drastically reduced the incidence of this particular etiology, so that Rhesus isoimmunization currently represents the underlying cause for hydrops in no more than $10 \%$ of cases [1012]. ABO blood group isoimmunization also occurs, but only rarely. The predominant anti-A and anti-B antibodies are IgM antibodies that do not cross the placenta. Hydrops due to isoimmunization is termed immune hydrops. The remaining etiologies are all aggregated together under the designation nonimmune hydrops (NIH). NIH will be the focus of this review. 
NIH may occur due to any one of potential causes listed in Table 1. The frequencies of the different etiologies vary among the numerous studies attempting to categorize this disorder. While small sample sizes and stochastic distributions likely account for many of the differences among these studies, factors intrinsic to each study also likely play a role. The specific geographic location of the sample population may be an important factor. The incidence of NIH due to $\alpha$-thalassemia is substantially higher in Southeast Asian countries, so studies emanating from this region will show much higher rates of underlying hematologic disorders than studies from anywhere else in the world [13-16]. Similarly, the types of NIH vary with the gestational age at onset. Studies that focus on a specific
Table 1 Categories of NIH with common individual etiologies

\begin{tabular}{|c|c|}
\hline Cardiac & Placental \\
\hline Atrioventricular canal defect & Twin-twin transfusion syndrome \\
\hline Left heart hypoplasia & Placental chorangioma \\
\hline \multicolumn{2}{|l|}{ Right heart hypoplasia } \\
\hline Tetralogy of Fallot & Lysosomal storage disorders \\
\hline In utero closure of the foramen ovale & Mucopolysaccharidosis I \\
\hline In utero closure of the ductus arteriosus & Mucopolysaccharidosis IVA \\
\hline Ebstein anomaly & Mucopolysaccharidosis VII \\
\hline Supraventricular tachycardia & Mucolipidosis II \\
\hline Atrial flutter & GM1 gangliosidosis \\
\hline \multirow[t]{2}{*}{ Wolf-Parkinson-White syndrome } & Sialidosis \\
\hline & Galactosialidosis \\
\hline Chromosomal & Gaucher disease \\
\hline Monosomy X & Niemann-Pick type A \\
\hline Trisomy 21 & Niemann-Pick type C \\
\hline Trisomy 18 & Infantile free sialic acid storage \\
\hline Trisomy 13 & Multiple sulfatase deficiency \\
\hline \multirow[t]{2}{*}{ Triploidy } & Farber disease \\
\hline & Wolman disease \\
\hline \multicolumn{2}{|l|}{ Syndromic } \\
\hline Noonan and Noonan-like & Thoracic \\
\hline Multiple pterygium syndrome & Congenital pulmonary airway malformation \\
\hline Campomelic dysplasia & Bronchopulmonary sequestration \\
\hline Achondroplasia & Diaphragmatic hernia \\
\hline Achondrogenesis & Chylothorax \\
\hline \multicolumn{2}{|l|}{ Osteogenesis imperfecta } \\
\hline Thanatophoric dysplasia & Gastrointestinal \\
\hline \multirow[t]{2}{*}{ Short-rib polydactyly syndrome } & Meconium peritonitis \\
\hline & Midgut volvulus \\
\hline Infectious & Intestinal malrotation \\
\hline Parvovirus B19 & Hepatic cirrhosis \\
\hline Cytomegalovirus & Hepatic necrosis \\
\hline Herpes simplex virus & Biliary atresia \\
\hline \multicolumn{2}{|l|}{ Syphilis } \\
\hline \multirow[t]{2}{*}{ Toxoplasma } & Urinary tract malformations \\
\hline & Urethral obstruction \\
\hline \multicolumn{2}{|l|}{ Hematologic } \\
\hline Alpha thalassemia & Tumors \\
\hline Glucose-6-phosphate dehydrogenase deficiency & Teratoma \\
\hline Erythrocyte pyruvate kinase deficiency & Lymphangioma \\
\hline Kasabach-Merritt Syndrome & Hemangioma \\
\hline Fetal hemorrhage & Neuroblastoma \\
\hline Fetomaternal hemorrhage & \\
\hline
\end{tabular}


trimester will show different frequencies of NIH from studies including all patients. Second trimester cases, for example, are more likely to have resulted from a chromosomal abnormality than are third trimester cases [7, 12]. Lastly, case ascertainment can bias the findings of these studies. Studies of cases detected on second trimester ultrasound will differ from those assessing live born hydropic NICU babies [11, 17]. It is also necessary to recognize that categories often overlap, so that one NIH case may fit into more than one category. Many karyotypically-abnormal hydropic fetuses also manifest structural cardiac disease, allowing the case to fit into either or both of these categories. Moreover, as prenatal diagnostic techniques continue to improve, it is likely that increased numbers of serious diseases will be identified prior to the onset of hydrops. The possibility of early treatment or termination for these cases may alter the distribution of hydrops cases that ultimately present to the pathologist.

\section{Specific Etiologies for NIH}

Despite the many caveats in the previous section, rough estimates for the different etiologies of NIH can be calculated. In general, cardiovascular causes are considered to be the most common among cases of NIH. Two large recent studies each, found that a little more than $20 \%$ of the cases were due to cardiovascular causes [11, 17, 18]. These cardiovascular etiologies may derive either from an intrinsic cardiac abnormality or from an external anomaly that leads to high output cardiac failure [5, 6]. Relevant external anomalies include vascular malformations or large, vascular tumors [5, 8]. A considerable number of structural cardiac anomalies have been associated with NIH [19]. Among these, the more common include atrioventricular canal defect, left heart hypoplasia, right heart hypoplasia, tetralogy of Fallot, in utero closure of the foramen ovale or ductus arteriosus, and Ebstein anomaly $[5,6,8,11]$. Rhythm disturbances are another frequent cardiac cause of NIH. Most of these are tachyarrhythmias, with supraventricular tachycardia, atrial flutter, and WolfParkinson-White syndrome being the most common [5, 6, 11]. Bradyarrhythmias can also be found. Many of these bradyarrythmias are due to destruction of the fetal conduction system by transplacental transmission of maternal autoantibodies $[5,6]$.

Chromosomal abnormalities also account for a significant percentage of NIH cases- $12.5 \%$ in one large retrospective literature review [17] and $7.5 \%$ in one large review of NICU NIH admissions [11]. Monosomy X (Turner Syndrome) is the most frequent chromosomal aberration found in most studies $[7,11,17,20]$, with trisomy 21 following a close second. The presence of congenital heart defects and/or transient abnormal myelopoiesis likely contribute to the high proportion of trisomy 21 cases with NIH. Trisomy 18, trisomy 13, and triploidy are also found consistently in NIH $[5,6]$. Other karyotypic abnormalities have been identified but are much less common. While the pathogenesis of NIH may be suggested by structural abnormalities (cardiac defects in trisomy 21 or monosomy $\mathrm{X}$, lymphatic dysplasia with chylothorax in monosomy $\mathrm{X}$ ) or hematologic abnormalities (transient abnormal myelopoiesis in trisomy 21), such anomalies are not always present in these cases. It seems likely that, in a subset of chromosomally abnormal fetuses with NIH, the hydrops arises through unclear mechanisms not readily identified at autopsy.

Closely related to chromosomal abnormalities are syndromic entities. The overall incidence of syndromic cases of NIH is approximately $5 \%$ [17]. Individual studies show highly variable incidence estimates, however [7]. Noonan and Noonan-like syndromes are among the more common syndromes associated with NIH [17, 21-23]. Disorders of restricted movement such as multiple pterygium syndrome may cause NIH, as may a number of skeletal dysplasias [5, 6]. Noonan and Noonan-like syndromes develop NIH primarily through the presence of cardiac defects and possibly lymphatic dysfunction. Although pathogenic mechanisms have been hypothesized for disorders of restricted movement or skeletal dysplasias, the mechanisms, in fact, remain unclear.

Infectious agents appear to cause approximately $7 \%$ of NIH cases $[11,17]$. Most of these organisms are viruses, with parvovirus B19 occurring at, by far, the greatest frequency. The in utero tropism of this virus for fetal granulocyte precursors (most importantly, erythroid precursors) leads to severe anemia, and its tropism for myocardium results in concomitant myocarditis and cardiac failure [5]. Together, these effects of parvovirus B19 infection can swiftly lead to NIH. Cytomegalovirus (CMV) is the second most frequent infectious cause of NIH, resulting in $1 \%$ of cases or more in most studies. Other viruses, such as herpes simplex virus (HSV), coxsackie viruses or adenoviruses occur much less frequently $[5,6,24]$. In addition, both the parasite toxoplasma and the spirochete Treponema pallidum are widely recognized as potential causes of NIH. As with parvovirus, the pathogenesis of NIH that develops in other infections is thought to result from a combination of anemia, endothelial damage with capillary leakage and myocardial damage. It seems possible that other, as yet undefined mechanisms may also contribute.

Hematologic disorders are another large category of NIH. These may result from increased loss of erythrocytes (due either to hemolysis or fetal hemorrhage) or decreased erythrocyte production [25]. Alpha-thalassemia is one of the more common causes of erythrocyte hemolysis. The 
incidence of this disorder is highly population dependent, though, with most cases occurring in those of Southeast Asian ancestry. Erythrocyte enzyme disorders, such as glucose-6-phosphate dehydrogenase deficiency and erythrocyte pyruvate kinase deficiency may also lead to NIH through erythrocyte hemolysis [26-28]. The microangiopathic hemolytic anemia seen in Kasabach-Merritt syndrome has been reported to result in NIH [29]. The fetus may also lose erythrocytes through a large but nonfatal fetomaternal hemorrhage [30, 31]. Tumors such as transient abnormal myelopoiesis, congenital leukemia, sacrococcygeal teratoma, and neuroblastoma may cause NIH by occupying the space needed for erythrocyte progenitor proliferation and maturation.

Placental abnormalities lead to 5-9 \% of NIH cases [11, 17]. Two conditions, monochorionic twinning and placental chorangioma, account for nearly all of these cases. Most if not all, monochorionic twin placentas are thought to contain vascular anastomoses between the twin circulations. In $10-15 \%$ of cases, these anastomoses create an imbalanced flow of blood between the twins leading to twin-twin transfusion syndrome (TTTS) [32]. TTTS can progress to the development of hydrops in either the donor or recipient twin. In fact, the appearance of hydrops in a twin is a criterion in the Quintero clinical staging system for TTTS [33]. Placental chorangiomas are highly vascular tumors that arise within the parenchyma of the placenta. Small chorangiomas $(<1 \mathrm{~cm})$ are quite common, while those larger than $>5 \mathrm{~cm}$ are much less frequent. Large chorangiomas can lead to NIH due to high output cardiac failure.

Lysosomal storage disorders have, until recently, been thought to cause relatively few cases of NIH. The largest systematic review of NIH found that only $1.1 \%$ of cases could be definitively ascribed to an inborn error of metabolism [17], and a large review of NICU NIH admissions found an even lower percentage at $0.8 \%$ [11]. However, these small percentages likely underestimate the true incidence of metabolic storage disorders in NIH [34, 35]. A recent meta-analysis found that, among cases of NIH tested for lysosomal storage disorders, $5.3 \%$ were positive, as were $17 \%$ of NIH cases initially classified as idiopathic [35].

Lesions that constrict the intrathoracic space, causing decreased venous return, are associated with $5 \%$ of NIH cases [17]. Constriction is often due to a large intrathoracic mass, such as a congenital pulmonary airway malformation (CPAM), diaphragmatic hernia, bronchopulmonary sequestration, or skeletal dysplasia [5, 6]. Large serous or chylous effusions frequently also lead to NIH.

A number of other, rare etiologies for NIH have also been identified. These include gastrointestinal obstructive lesions, such as meconium peritonitis or midgut volvulus; hepatic disorders, including hepatic cirrhosis or necrosis and biliary atresia; urinary tract malformations (primarily bladder outlet obstruction); and multiple extrathoracic tumors [5, 6, 17]. Importantly, despite extensive evaluation, a surprisingly high number of NIH cases remain idiopathic—at least $15 \%$ [6, 17].

\section{Prenatal Workup of Hydrops}

Currently, fetal hydrops is nearly always diagnosed prenatally during ultrasound examination of the fetus. The finding of hydrops at this stage initiates a clinical evaluation to identify the etiology for the hydrops. Establishing the etiology is important for counseling the parents about the fetal prognosis, for detecting any disorders amenable to in utero fetal therapy and for determining the timing of delivery. The Society for Maternal-Fetal Medicine has recently published new guidelines to aid in the selection of appropriate prenatal testing [6]. This testing begins with a thorough family history to detect any maternal illnesses, to identify any familial genetic disorders and to seek out evidence for consanguinity. An extensive fetal ultrasound examination is performed to seek any structural anomalies that may have contributed to the development of hydrops. This ultrasound examination should include an echocardiogram since cardiac anomalies are one of the most frequent underlying causes of fetal hydrops. Also part of the ultrasound examination, particularly if the anatomical survey is otherwise unremarkable, is evaluation of the blood flow in the middle cerebral artery (MCA) since fetal anemia typically manifests as an increase in the peak systolic velocity of the MCA flow.

The results of the ultrasound examination then guide subsequent testing, dividing the cases into those with or without anomalies. For cases with structural abnormalities, the initial assumption is often made that these anomalies are etiologically related to the hydrops. In this situation, either amniocentesis or fetal blood sampling is recommended to acquire fetal tissue for testing. Karyotype or, preferably, chromosomal microarray analysis is recommended. Microarray can detect all abnormal chromosomal regions visible by karyotype analysis except for balanced translocations, which are unlikely to be relevant in the hydropic fetus. Importantly, microarray can detect much smaller anomalies than can karyotype, and the cost of microarray analysis has dropped near to the level of a karyotype (although there currently remain issues with insurance coverage for microarray studies in the US) [36]. If the ultrasound findings are suspicious for a fetal infection, then PCR testing for CMV and toxoplasma are recommended on the amniotic fluid or fetal blood [6].

For those cases with no anomalies identified, maternal blood is tested for alloimmunization. The maternal blood 
type (particularly the Rhesus antigen) is rechecked, and an indirect Coombs test is performed. A maternal complete blood count is recommended. Either a Kleihauer-Betke test or flow cytometry should be carried out to detect a significant fetomaternal hemorrhage. Serologic testing for parvovirus B19 is recommended, as is serologic testing for syphilis [6].

The cases with no ultrasound anomalies are then further divided by the results of the MCA Doppler studies. Those cases with elevated peak systolic velocities in the MCA are worked up for fetal anemia. The maternal mean corpuscular volume (MCV) from the complete blood count should be evaluated, since a low MCV is suggestive of $\alpha$ thalassemia. Fetal tissue should be obtained via amniocentesis or fetal blood sampling. Karyotype or microarray should be performed. PCR studies for parvovirus B19 should be carried out. PCR studies to detect CMV or toxoplasma are also recommended. Additional studies to identify rare entities, such as glucose-6-phosphate dehydrogenase deficiency (G-6-PD) may also be performed [6].

For those cases with a normal MCA Doppler study, amniocentesis or fetal blood sampling is again recommended so that karyotype or microarray can be performed. PCR studies to detect CMV and toxoplasma are again recommended. In addition, for any of the above situations, lysosomal enzyme testing may be initiated [6].

\section{Autopsy Examination for Hydrops}

The anatomic pathologist is generally consulted in cases either when the fetus is terminated or the fetus or neonate has died. For many, the clinician will have already made a presumptive diagnosis for the underlying etiology. For the remainder, determining the cause of the NIH will be the primary question at autopsy. In either case, it is important to approach the autopsy with an open mind to ensure that clinical suspicions do not unduly bias the pathologist and do not deter him or her from rigorously performing all appropriate tests. That said, one of the first tasks for the pathologist is to gather and assess all available clinical information. This process includes determining which laboratory tests have already been done, and then attempting to obtain those that remain. Maternal testing such as a Kleihauer-Betke test for fetomaternal hemorrhage or a complete blood count with MCV provides invaluable information that may be difficult, if not impossible, to obtain at autopsy alone. Similarly, if permission for amniocentesis or fetal blood sampling was denied during the pregnancy, then all recommended prenatal testing on fetal samples (such as microarray or PCR studies) will need to be done on autopsy tissues. Of note, prenatal cell-free fetal DNA testing (otherwise termed noninvasive prenatal testing) is rapidly expanding in many countries. Currently, caution would suggest considering this as a screening test requiring confirmation by karyotype or microarray.

After assessing the extent of the prenatal testing and mapping out potential additional tests, the autopsy can begin. Excellent comprehensive reviews covering autopsy examination in stillbirth (including hydrops specifically) have recently been published [5, 37]. The specifics of these detailed protocols are beyond the scope of this brief review. Instead, familiarity with the basics of the perinatal autopsy will be assumed, and areas of particular importance will be highlighted. It is also important to note that the extensive workup outlined below is most relevant to a pathology practice in a developed country with generous hospital resources for autopsy. The procedure must, of course, be modified by the availability of resources for any given case.

Prior to any incisions, the body should be photographed extensively. These photographs must include close-up views of the face, profile, hands, feet and any anomalies. Careful full-body radiographs should also be performed. Full body radiographs are an underutilized modality both for the examination of stillbirths in general and for the evaluation of the hydropic fetus or neonate [38], and interpretation of the photographs and radiographs by a pediatric geneticist may provide diagnostic information or help in choosing ancillary studies. Minimally-invasive autopsy is not recommended for the hydropic fetus or neonate unless there is no alternative. Although these autopsies have proven useful, particularly when the parents wish to avoid a full autopsy [39], the limited options for obtaining tissue for ancillary studies and the inability to microscopically examine the tissues argue against this technique.

Sampling of body fluids and tissues during a hydrops autopsy should be extensive. Portions of liver, skin, heart and brain should be snap frozen. Pleural fluid should be collected to help identify chylothorax. Blood should be collected and a blood card spotted. Urine should also be collected and frozen. The small amount of blood and urine available from a fetus or neonate at autopsy significantly limits the number of studies that can be performed on these fluids, however. This limitation highlights the importance of fibroblast cultures as an alternative source of fetal tissue. Cultured fetal cells are an excellent resource for DNA extraction or for performing enzymatic tests, particularly those for lysosomal storage disorders. Multiple tissues should be sampled for fibroblast cultures to ensure their growth and availability. Fetal cells can grow from skin, fascia or fetal cartilage [5]. In cases of moderate to severe maceration, placental tissue (chorionic villi and umbilical cord) should also be sent for tissue culture. Placental 
tissues will often grow in culture long after fetal body cells have lost all vitality. While having these cultured cells available for testing may be essential to identify the etiology in a case of $\mathrm{NIH}$, extensive testing for lysosomal storage disorders should probably occur only after initial autopsy examination and testing fails to yield a clear diagnosis.

Histologic examination of the hydropic fetus or neonate begins with the placenta. The hydropic placenta often appears edematous and may demonstrate delayed villous maturation. Erythroid precursors may be increased within the fetal circulation, suggesting a fetal anemia. The infectious agents that can cause NIH may be detected within the placenta. The viral inclusions of CMV and HSV may be found, particularly in the context of a lymphoplasmacytic chronic villitis. Parvoviral inclusions may be seen within fetal erythroid precursors in the fetal vessels. Although the placenta in parvovirus infection may seem cellular, this appearance usually results more from endothelial cell reactivity than from chronic villitis. Chronic villitis with a plasma cell component often accompanies both toxoplasma and Treponema pallidum. Finally, a subset of lysosomal storage disorders presents with impressive accumulations of material within trophoblast and Hofbauer cells.

Histologic examination of body tissues can be guided, at least in part, by the anatomic findings. While thorough sampling of organs is generally recommended, a previous definitive diagnosis may allow truncation of the sampling. Cardiac cases may benefit from histologic examination of the cardiac conduction system. In cases of movement disorders, fetal CNS (with spinal cord), skeletal muscle, and skin should be carefully examined. Skeletal dysplasias require extensive sampling of bones, particularly along the costochondral junction. The liver can be a remarkably informative organ for multiple entities. Infections often involve the liver, where they can be readily identified by histology or immunohistochemistry. Hemolytic processes may result in significant iron deposition in the liver. The amount and cell composition of the extramedullary hematopoiesis in the liver may suggest anemia. Storage disorders often lead to accumulations of material within the liver. Such accumulations also often develop in the spleen and bone marrow.

Current recommendations for genetic testing in cases of NIH include microarray and any specifically indicated DNA sequence analyses. These recommendations are likely to change in the relatively near future as various next-generation sequencing (NGS) technologies emerge and become commercially viable [36]. These technologies rely on multiple short reads of DNA sequence that are subsequently stitched together by computer algorithms. The extensive computer analysis is a major source of the cost for these analyses. NGS panels are currently being developed for various disorders (including hydrops [17]). These panels minimize the cost by focusing on a small set of sequences and discarding the rest from the analysis. As costs for NGS continue to decrease, less-biased strategies such as whole-exome sequencing or whole-genome sequencing (which strive to sequence all-protein coding regions and the entire genome, respectively) may ultimately be employed.

\section{Conclusion}

NIH represents the near terminal decompensation of a fetus afflicted by one of a strikingly large number of possible diseases. Autopsy examination with ancillary testing remains an important tool for identifying the underlying etiology of NIH, particularly in those cases where no diagnosis could be established prenatally.

\section{Compliance with Ethical Standards}

Conflict of interest None.

\section{References}

1. Skoll MA, Sharland GK, Allan LD. Is the ultrasound definition of fluid collections in non-immune hydrops fetalis helpful in defining the underlying cause or predicting outcome? Ultrasound Obstet Gynecol. 1991;1(5):309-12.

2. Bellini C, Hennekam RC. Non-immune hydrops fetalis: a short review of etiology and pathophysiology. Am J Med Genet A. 2012;158A(3):597-605.

3. Lee AJ, Bethune M, Hiscock RJ. Placental thickness in the second trimester: a pilot study to determine the normal range. J Ultrasound Med. 2012;31(2):213-8.

4. Hoddick WK, Mahony BS, Callen PW, Filly RA. Placental thickness. J Ultrasound Med. 1985;4(9):479-82.

5. Faye-Petersen OM, Heller DS. Pathology of the stillborn infant for the general pathologist: part 2. Adv Anat Pathol. 2015;22(2):71-93.

6. Society for Maternal-Fetal Medicine (SMFM), Norton ME, Chauhan SP, Dashe JS. Society for maternal-fetal medicine (SMFM) clinical guideline \#7: nonimmune hydrops fetalis. Am J Obstet Gynecol. 2015;212(2):127-39.

7. Heinonen S, Ryynänen M, Kirkinen P. Etiology and outcome of second trimester non-immunologic fetal hydrops. Acta Obstet Gynecol Scand. 2000;79(1):15-8.

8. Machin GA. Hydrops revisited: literature review of 1,414 cases published in the 1980s. Am J Med Genet. 1989;34(3):366-90.

9. Hutchison AA, Drew JH, Yu VY, Williams ML, Fortune DW, Beischer NA. Nonimmunologic hydrops fetalis: a review of 61 cases. Obstet Gynecol. 1982;59(3):347-52.

10. Santolaya J, Alley D, Jaffe R, Warsof SL. Antenatal classification of hydrops fetalis. Obstet Gynecol. 1992;79(2):256-9.

11. Abrams ME, Meredith KS, Kinnard P, Clark RH. Hydrops fetalis: a retrospective review of cases reported to a large national database and identification of risk factors associated with death. Pediatrics. 2007;120(1):84-9.

12. Sohan K, Carroll SG, De La Fuente S, Soothill P, Kyle P. Analysis of outcome in hydrops fetalis in relation to gestational 
age at diagnosis, cause and treatment. Acta Obstet Gynecol Scand. 2001;80(8):726-30.

13. Suwanrath-Kengpol C, Kor-anantakul O, Suntharasaj T, Leetanaporn R. Etiology and outcome of non-immune hydrops fetalis in southern Thailand. Gynecol Obstet Invest. 2005;59(3):134-7.

14. Liao C, Wei J, Li Q, Li J, Li L, Li D. Nonimmune hydrops fetalis diagnosed during the second half of pregnancy in Southern China. Fetal Diagn Ther. 2007;22(4):302-5.

15. Ng ZM, Seet MJ, Erng MN, Buendia F, Chang AS, Sriram B. Nonimmune hydrops fetalis in a children's hospital: a six-year series. Singapore Med J. 2013;54(9):487-90.

16. Yeom W, Paik ES, An JJ, Oh SY, Choi SJ, Roh CR, et al. Clinical characteristics and perinatal outcome of fetal hydrops. Obstet Gynecol Sci. 2015;58(2):90-7.

17. Bellini C, Donarini G, Paladini D, Calevo MG, Bellini T, Ramenghi LA, et al. Etiology of non-immune hydrops fetalis: an update. Am J Med Genet A. 2015;167(5):1082-8.

18. Bellini C, Hennekam RC, Fulcheri E, Rutigliani M, Morcaldi G, Boccardo F, et al. Etiology of nonimmune hydrops fetalis: a systematic review. Am J Med Genet A. 2009;149A(5):844-51.

19. Knilans TK. Cardiac abnormalities associated with hydrops fetalis. Semin Perinatol. 1995;19(6):483-92.

20. Santo S, Mansour S, Thilaganathan B, Homfray T, Papageorghiou A, Calvert S, et al. Prenatal diagnosis of non-immune hydrops fetalis: what do we tell the parents? Prenat Diagn. 2011;31(2):186-95.

21. Croonen EA, Nillesen WM, Stuurman KE, Oudesluijs G, van de Laar IM, Martens L, et al. Prenatal diagnostic testing of the Noonan syndrome genes in fetuses with abnormal ultrasound findings. Eur J Hum Genet. 2013;21(9):936-42.

22. Gargano G, Guidotti I, Balestri E, Vagnarelli F, Rosato S, Comitini G, et al. Hydrops fetalis in a preterm newborn heterozygous for the c. $4 \mathrm{~A}>\mathrm{G}$ SHOC2 mutation. Am J Med Genet A. 2014;164A(4):1015-20.

23. Lee KA, Williams B, Roza K, Ferguson H, David K, Eddleman $\mathrm{K}$, et al. PTPN11 analysis for the prenatal diagnosis of Noonan syndrome in fetuses with abnormal ultrasound findings. Clin Genet. 2009;75(2):190-4.

24. Barron SD, Pass RF. Infectious causes of hydrops fetalis. Semin Perinatol. 1995;19(6):493-501.

25. Arcasoy MO, Gallagher PG. Hematologic disorders and nonimmune hydrops fetalis. Semin Perinatol. 1995;19(6):502-15.

26. Perkins RP. Hydrops fetalis and stillbirth in a male glucose-6phosphate dehydrogenase-deficient fetus possibly due to maternal ingestion of sulfisoxazole; a case report. Am J Obstet Gynecol. 1971;111(3):379-81.

27. Ferreira P, Morais L, Costa R, Resende C, Dias CP, Araújo F, et al. Hydrops fetalis associated with erythrocyte pyruvate kinase deficiency. Eur J Pediatr. 2000;159(7):481-2.

28. Hennekam RC, Beemer FA, Cats BP, Jansen G, Staal GE. Hydrops fetalis associated with red cell pyruvate kinase deficiency. Genet Couns. 1990;1(1):75-9.

29. Skopec LL, Lakatua DJ. Non-immune fetal hydrops with hepatic hemangioendothelioma and Kasabach-Merritt syndrome: a case report. Pediatr Pathol. 1989;9(1):87-93.

30. Fay RA. Feto-maternal haemorrhage as a cause of fetal morbidity and mortality. Br J Obstet Gynaecol. 1983;90(5):443-6.

31. Cardwell MS. Successful treatment of hydrops fetalis caused by fetomaternal hemorrhage: a case report. Am J Obstet Gynecol. 1988;158(1):131-2.

32. Blickstein I. Monochorionicity in perspective. Ultrasound Obstet Gynecol. 2006;27(3):235-8.

33. Quintero RA, Morales WJ, Allen MH, Bornick PW, Johnson PK, Kruger M. Staging of twin-twin transfusion syndrome. J Perinatol. 1999;19(8 Pt 1):550-5.

34. Whybra C, Mengel E, Russo A, Bahlmann F, Kampmann C, Beck M, et al. Lysosomal storage disorder in non-immunological hydrops fetalis (NIHF): more common than assumed? Report of four cases with transient NIHF and a review of the literature. Orphanet J Rare Dis. 2012;7:86.

35. Gimovsky AC, Luzi P, Berghella V. Berghella, Lysosomal storage disease as an etiology of nonimmune hydrops. Am J Obstet Gynecol. 2015;212(3):281-90.

36. Peters DG, Yatsenko SA, Surti U, Rajkovic A. Recent advances of genomic testing in perinatal medicine. Semin Perinatol. 2015;39(1):44-54.

37. Heller DS, Faye-Petersen OM. Pathology of the stillborn infant for the general pathologist: part 1. Adv Anat Pathol. 2015;22(1): 1-28.

38. Swenson E, Schema L, McPherson E. Radiographic evaluation of stillbirth: what does it contribute? Am J Med Genet A. 2014;164A(9):2270-5.

39. Whitby $\mathrm{EH}$, Offiah $\mathrm{AC}$, Cohen MC. Initial experiences of a minimally invasive autopsy service. A report of the techniques and observations in the first 11 cases. Pediatr Dev Pathol. 2015;18(1):24-9. 